\title{
Dissipative Dynamics and Uncertainty Measures of a Charged Oscillator in the Presence of the Aharonov-Bohm Effect
}

\author{
J. P. G. Nascimento*1, V. Aguiar ${ }^{10}$, D. S. Cortez ${ }^{1}$, I. Guedes ${ }^{1}$ \\ ${ }^{1}$ Universidade Federal do Ceará, Departamento de Física, 60455-760, Fortaleza, CE, Brasil.
}

Received on November 02, 2020. Accepted on December 01, 2020.

\begin{abstract}
We analyze the effects of dissipation in a charged oscillator in the presence of the Aharonov-Bohm effect by using time-dependent mass $(m(t))$ Hamiltonians. We consider two different models for the dissipative Hamiltonian and analyze the uncertainties $(\Delta \boldsymbol{r}$ and $\Delta \boldsymbol{p})$ and the quantum mechanical expectation value of energy $(\langle E\rangle)$ in terms of time $(t)$, damping parameters and flux parameter $(v)$. For the Caldirola-Kanai model, we observe that the flux parameter $v$ decreases the energy dissipation in a quantum dot for a certain range of $t$.

Keywords: Aharanov-Bohm effect, Caldirola-Kanai oscillators, Lane-Endem oscillators, uncertainty, mean energy, 2D parabolic quantum dot.
\end{abstract}

\section{Introduction}

Since the 1920s, the interaction between charged particles and electromagnetic fields has attracted a great attention in the literature under the quantum mechanics point of view. Surprisingly, the particle motion can sometimes be influenced by electromagnetic fields in regions where both magnetic field $\boldsymbol{B}$ and electric field $\boldsymbol{E}$ are zero. As a matter of fact, the particle is affected by electromagnetic potentials, which may exist in regions where the fields do not exist. This effect was first pointed out in 1949 by Ehrenburg and Siday [1]. Using a semiclassical approach, they predicted a fringe shift due to magnetic vector potentials in a field-free region. Later, in 1959, Aharonov and Bohm [2] discussed the role of the electromagnetic potentials in quantum mechanics. In the field-free multiply connected regions, the results of interference and scattering experiments depend on integrals of the potentials. This phenomenon has come to be called the Aharonov-Bohm (AB) effect.

Over the years, the AB effect has been experimentally verified [3-5] becoming a very interesting research area. The AB effect has been studied in several mesoscopic systems, such as metal rings [6], carbon nanotubes [7, 8] and monolayer [9-12] and bilayer [13] graphene. Ferkous and Bounames [14] obtained the energy spectrum and the eigenfunctions of a $2 D$ Dirac oscillator in the presence of $\mathrm{AB}$ effect. They showed that the energies depend on the particle spin and the $\mathrm{AB}$ magnetic flux parameter. Bouguerra, Maamache and Bounames [15] using the invariant operator method [16] obtained the exact wave-functions of a time-dependent $2 D$ harmonic

\footnotetext{
${ }^{*}$ Correspondence email address: joaopedro@fisica.ufc.br.
}

oscillator in the presence of the AB effect. They analyzed the case of a very thin solenoid (a flux tube of zero radius $[17-19])$. The $\mathrm{AB}$ effect in presence of dissipation has also been investigated [20, 21]. Guinea [20] obtained the amplitude of the $\mathrm{AB}$ oscillations of a particle on a ring threaded by a magnetic flux and coupled to different dissipative environments. One of these environments is the Caldeira-Leggett harmonic bath model 22 .

The Caldeira-Leggett model is a fundamental approach in which the system is coupled to a harmonic bath (a collection of harmonic oscillators) having many degrees of freedom. The energy flows from the system to the bath. However, there exists other approaches used to introduce dissipation in quantum mechanics [23 28]. Here we consider a phenomenological description which includes dissipation by means of explicitly time-dependent Hamiltonians 25 28. This phenomenological approach is based on the so-called Caldirola-Kanai (CK) [25, 26] Hamiltonian, given by

$$
H(t)=e^{-\int \gamma(t) d t} \frac{p^{2}}{2 m_{0}}+e^{\int \gamma(t) d t} V(x),
$$

where $x$ and $p$ are position and momentum coordinates, respectively, and $\gamma(t)$ is the damping coefficient. This model has already been used to study dissipative quantum tunneling [29, 30, time-dependent mesoscopic RLC circuits [31 35], damped effects on the entanglement of a two-level atom in a two-photon field [36, dissipative relativistic motion [37, the matter black-body problem [38, parabolic confined particles (dissipative quantum dots) [39, 40, and the dynamics of the DNA breathing 41. The full correspondence between the system-reservoir approach and time-dependent Hamiltonians has been shown by Yun and Sun [42, 43. 
In this work we follow the procedure by Bouguerra, Maamache and Bounames [15] to study the effects of dissipation in a charged oscillator in the presence of the AB effect. This paper is organized as follows. In Section 2, we briefly explain the fundamental definitions needed for the calculations. In Section 3 we calculate the uncertainties and energy to both CK and Lane-Emden (LE) dissipative systems and summarize the results.

\section{Basic Definitions}

Consider the time-dependent Hamiltonian given by

$$
H(t)=\frac{(\boldsymbol{p}-e \boldsymbol{A})^{2}}{2 M(t)}+\frac{1}{2} M(t) \omega^{2}(t)\left(x^{2}+y^{2}\right),
$$

where $\boldsymbol{A}$ is the potential vector. Following Refs. [15, 17 19], for a flux tube of zero radius the corresponding potential $\boldsymbol{A}$ in the Coulomb gauge is given by

$$
e \boldsymbol{A}=\nu\left(\frac{y}{x^{2}+y^{2}},-\frac{x}{x^{2}+y^{2}}, 0\right),
$$

where $\nu$ is a finite nonzero flux parameter. The timedependent Schrödinger equation for the Hamiltonian (2) with potential vector (3) reads

$$
\begin{aligned}
i \hbar \frac{\partial}{\partial t} \psi_{n}(\boldsymbol{r}, t)= & {\left[\frac{\boldsymbol{p}^{2}}{2 M(t)}+\frac{1}{2} M(t) \omega^{2}\left(x^{2}+y^{2}\right)\right.} \\
& \left.+\frac{\left(2 \nu L_{z}+\nu^{2}\right)}{2 M(t)\left(x^{2}+y^{2}\right)}\right] \psi_{n}(\boldsymbol{r}, t),
\end{aligned}
$$

and $L_{z}=x p_{y}-y p_{x}$ is the angular momentum in the $\widehat{\boldsymbol{z}}$ direction.

According to Ref. [15 the solution of the timedependent Schrödinger equation (4) reads

$$
\begin{aligned}
\psi_{n, m}(x, y, t)= & \frac{C_{n, m}}{\rho} e^{i m \theta} \\
& \times \exp \left[-i\left(2 n+\left|m+\frac{\nu}{\hbar}\right|+1\right) \int_{0}^{t} \frac{1}{M \rho^{2}} d t^{\prime}\right] \\
& \times \exp \left[\left(x^{2}+y^{2}\right)\left(\frac{i M \dot{\rho}}{2 \hbar \rho}-\frac{1}{2 \hbar \rho^{2}}\right)\right] \\
& \times\left(x^{2}+y^{2}\right) \frac{\left|m+\frac{\nu}{\hbar}\right|}{\hbar} \\
& \times{ }_{1} F_{1}\left(-n,\left|m+\frac{\nu}{\hbar}\right|+1, \frac{x^{2}+y^{2}}{\hbar \rho^{2}}\right)
\end{aligned}
$$

where $C_{n, m}=\frac{1}{\Gamma\left(\left|m+\frac{\nu}{\hbar}\right|+1\right)}\left[\frac{\Gamma\left(n+\left|m+\frac{\nu}{\hbar}\right|+1\right)}{\left.n ! \pi \hbar\right|^{m+\frac{\nu}{\hbar} \mid+1}}\right]^{1 / 2}, \theta=$ $\tan ^{-1}\left(\frac{y}{x}\right),{ }_{1} F_{1}(a, b, c)$ denotes the confluent hypergeometric function of the first kind and $\rho(t)$ satisfies the generalized Milne-Pinney (MP) [44, 45] equation

$$
\ddot{\rho}+\frac{\dot{M}}{M} \dot{\rho}+\omega^{2} \rho=\frac{1}{M^{2}(t) \rho^{3}} .
$$

\section{Results and Discussion}

From Eq. (5) we write the following relations

$$
\begin{gathered}
\left\langle r^{2}\right\rangle_{n, m}=\hbar \rho^{2}\left(2 n+\left|m+\frac{\nu}{\hbar}\right|+1\right), \\
\langle x\rangle_{n, m}=\langle y\rangle_{n, m}=0, \\
\left\langle p^{2}\right\rangle_{0,0}=\hbar\left(\frac{|v|}{\hbar} M^{2} \dot{\rho}^{2}+\frac{1+M^{2} \rho^{2} \dot{\rho}^{2}}{\rho^{2}}\right), \\
\left\langle p_{x}\right\rangle_{0,0}=\left\langle p_{y}\right\rangle_{0,0}=0 .
\end{gathered}
$$

Equations (7)-10 are written in terms of $\rho$, a c-number quantity satisfying the generalized MP equation (see Eq. (6)). For a given $M(t)$ and $\omega(t)$ one has to solve Eq. (6) and consider only real solutions of $\rho$ so that $I(t)$ is Hermitian. Observe that the Hamiltonian (2) describes the dynamics of dissipation in a $2 \mathrm{D}$ parabolic quantum dot in the presence of a Aharanov-Bohm effect if $\omega(t)=\omega_{0}$.

Now let us consider the Caldirola-Kanai (CK) model, where $M(t)=m_{0} e^{\gamma t}$ and $\omega(t)=\omega_{0}$. In this case, the solution of the Milne-Pinney equation (6) is $\rho=\frac{1}{\sqrt{m_{0} \Omega}} e^{-\left(\frac{\gamma t}{2}\right)}\left(\right.$ with $\Omega=\sqrt{\omega_{0}^{2}-\frac{\gamma^{2}}{4}}>0$ ) [46]. In Figs. 1 (a)-(b), we show plots of the uncertainties in position (Fig. 1(a)) and momentum (Fig. 1(b)) spaces of the system in the ground state. We show that both $\Delta \boldsymbol{r}$ and $\Delta \boldsymbol{p}$ increase with increasing $\nu$. Figure 1 (a) also shows that $\Delta \boldsymbol{r}$ tends to zero with increasing time as an effect of the dissipation (remember that classically the position of a damped oscillator tends to zero with increasing time, leading to a minimum uncertainty in the position). For $v=0$, we observed that the particle's localization due to the CK dissipation is always lower than the uncertainty of a $2 \mathrm{D}$ parabolic quantum dot without dissipation and $\mathrm{AB}$ effect. On the other hand, for $v=5(v=10)$, the particle's delocalization is greater than that of a $2 \mathrm{D}$ parabolic quantum dot without dissipation and $\mathrm{AB}$ effect in the range $t<1.9(t<2.5)$.

From Eqs. (7) -10 we see that the uncertainty product $\Delta \boldsymbol{r} \Delta \boldsymbol{p}$ obeys the Heisenberg Uncertainty Principle $(\Delta \boldsymbol{r} \Delta \boldsymbol{p} \geq \hbar)$ and it does not depend on $t$. In Figs. 2(a)-(b) we plot $\Delta \boldsymbol{r} \Delta \boldsymbol{p}$ as function of $\gamma$ and $v$, respectively. We observe that $\Delta \boldsymbol{r} \Delta \boldsymbol{p}$ increases nonlinearly (linearly) with the increasing of $\gamma(v)$. Note also that $\Delta \boldsymbol{r} \Delta \boldsymbol{p}(\gamma)$ increases abruptly as $\gamma$ approaches to $\gamma \rightarrow 2 \omega_{0}$ in the limit $\Omega \rightarrow 0$.

Another class of damped system is the LE oscillator [47 50] where $M(t)=m_{0} t^{\alpha}$ and $\omega(t)=\omega_{0}$. In this system the damping coefficient depends on time, $\gamma(t)=\frac{\alpha}{t}$. For $\alpha=2$, the solution of the Milne-Pinney equation (6) is $\rho=\frac{t^{-1}}{\sqrt{m_{0} \omega_{0}}}$ [48]. In Figs. 3(a)-(b) we show plots comparing the uncertainties in position (Fig. 3(a)) and momentum (Fig. 3(b)) spaces for a $2 D$ quantum dot in the presence of the $\mathrm{AB}$ effect obtained from $\mathrm{CK}$ and LE dissipation in the ground state. For $t<2$, we observe that the decrease of $\Delta \boldsymbol{r}$ due to the LE dissipation is 
(a)

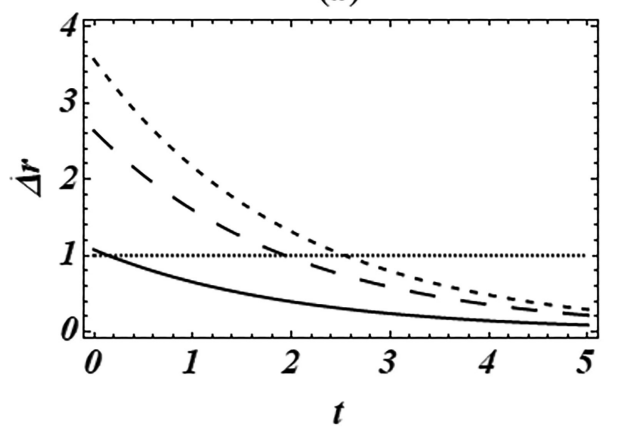

(b)

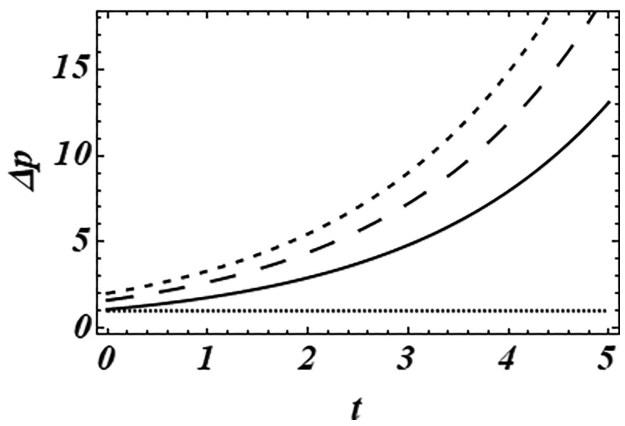

Figure 1: Plots of (a) $\Delta \boldsymbol{r}$ and (b) $\Delta \boldsymbol{p}$ for $v=0$ (solid line), $v=5$ (large dashed line) and $v=10$ (short dashed line) for the ground state of the system by considering the CK dissipation. The dotted line corresponds to the uncertainties of a 2D parabolic quantum dot without dissipation and $\mathrm{AB}$ effect, where $M(t)=m_{0}, \omega(t)=\omega_{0}$ and $v=0$. In this figure we use $\hbar=m_{0}=\omega_{0}=\gamma=1$.

(a)

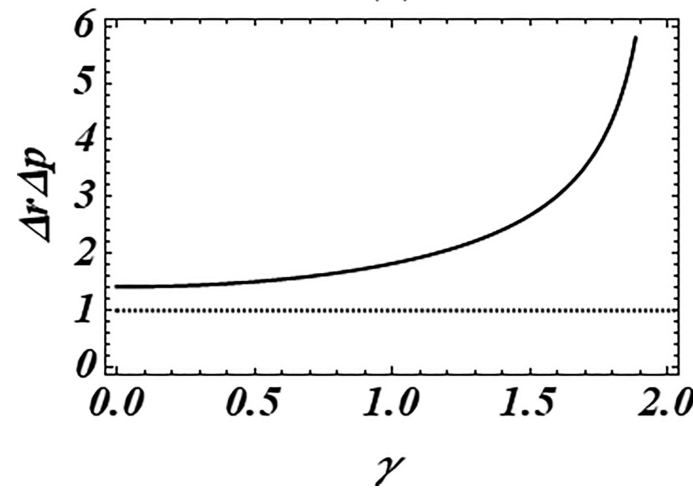

(b)

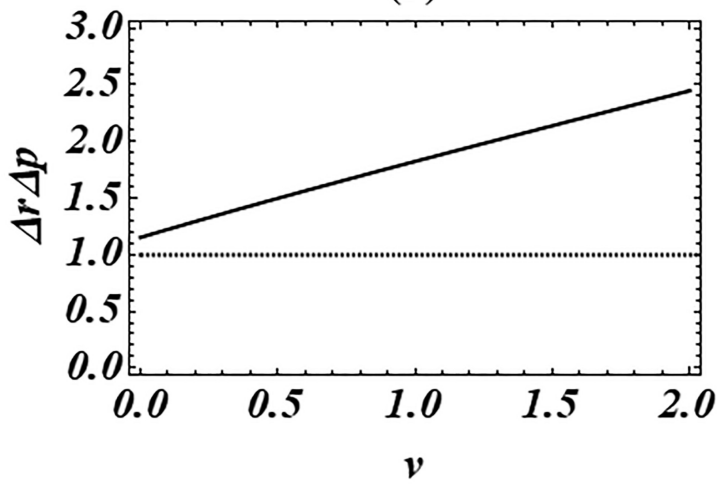

Figure 2: Plots of $\Delta \boldsymbol{r} \Delta \boldsymbol{p}$ as function of (a) $\gamma$ and (b) $v$ for the ground state of the system by considering the CK dissipation. In Figure 2(a) we keep $v=1$, while in Figure 2(b) $\gamma=1$. The dotted line corresponds to the uncertainties of a 2D parabolic quantum dot without dissipation and $\mathrm{AB}$ effect, where $M(t)=m_{0}, \omega(t)=\omega_{0}$ and $v=0$. In both figures we use $\hbar=m_{0}=\omega_{0}=t=1$.

(a)

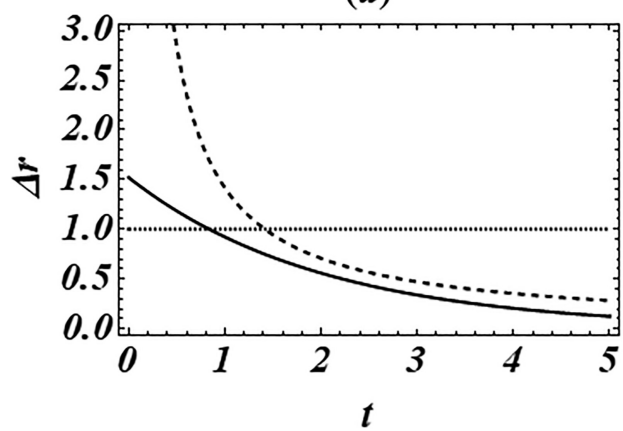

(b)

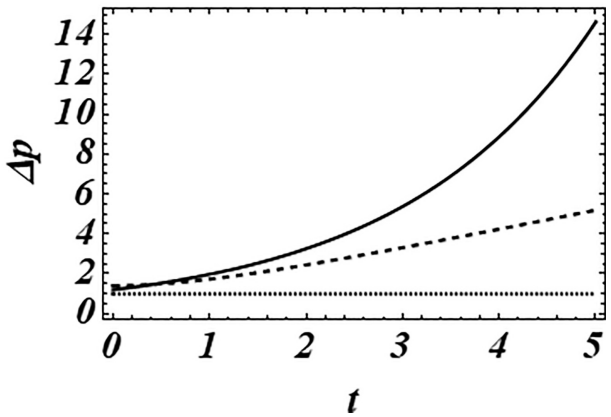

Figure 3: Plots of (a) $\Delta \boldsymbol{r}$ and (b) $\Delta \boldsymbol{p}$ for CK (solid line) and LE (dashed line) oscillators for the ground state of the system. The dotted line corresponds to the uncertainties of a $2 \mathrm{D}$ parabolic quantum dot without dissipation and $\mathrm{AB}$ effect, where $M(t)=m_{0}$, $\omega(t)=\omega_{0}$ and $v=0$. In this figure we use $v=\gamma=\hbar=m_{0}=\omega_{0}=1$.

steeper than that for the CK one. In this range, the LE damping coefficient is always greater than 1 and tends to $+\infty$ in the limit that $t \rightarrow 0$. We also observe that the particle is less localized due to LE dissipation for all values of $t$.

Using the procedure described by Hasse [27, the timedependent quantum mechanical expectation value of energy for the lowest-lying state is given by

$$
\begin{aligned}
\langle E\rangle_{0,0}= & \frac{\hbar m_{0}}{2 M(t)}(1+|v|) \\
& \times\left(\frac{1}{M(t) \rho^{2}(t)}+M(t) \dot{\rho}^{2}(t)+M(t) \omega_{0}^{2} \rho^{2}(t)\right) .
\end{aligned}
$$




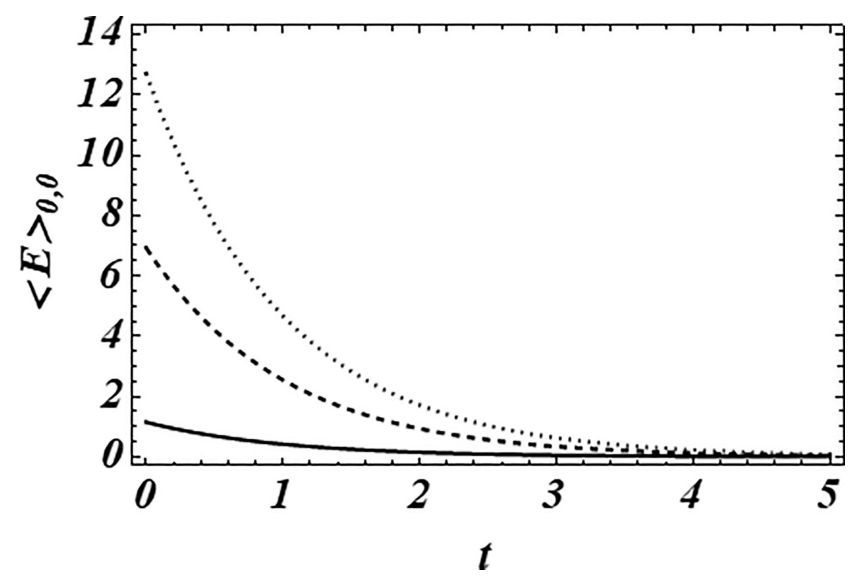

Figure 4: Plots of $\langle E\rangle_{0,0}$ for $v=0$ (solid line), $v=5$ (dashed line) and $v=10$ (dotted line) for the ground state of the system by considering the CK dissipation. In this figure we use $\hbar=$ $m_{0}=\omega_{0}=\gamma=1$.

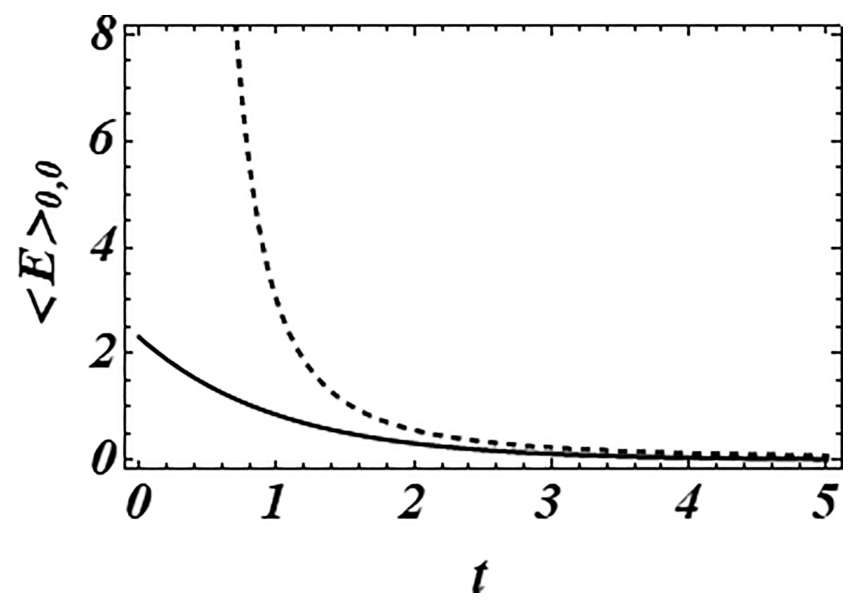

Figure 5: Plots of $\langle E\rangle_{0,0}$ for CK (solid line) and LE (dashed line) oscillators for the ground state of the system. In this figure we use $v=\gamma=\hbar=m_{0}=\omega_{0}=1$.

In Fig. 4 we show plots of $\langle E\rangle_{0,0}$ for different values of $\nu$ in a CK dissipative system. As expected, the energy decreases with increasing $t$. However, in the range $t<3.5 \mathrm{~s}$ we can minimize the dissipation in the $2 \mathrm{D}$ parabolic quantum dot by increasing $\nu$. In Fig. 5 we compare the energies for both CK and LE systems. We observe that for $t<2$ the LE dissipation is steeper than that of the CK one. This result is also due to the behavior of the LE damping coefficient in that range.

Summing up, we studied the effects of dissipation in a charged oscillator in the presence of the AB effect by using time-dependent mass $(m(t))$ Hamiltonians. The procedure used describes the dynamics of the dissipation in $2 \mathrm{D}$ parabolic quantum dot in the presence of $\mathrm{AB}$ effect. From the exact wavefunctions [15], we calculated the uncertainties $(\Delta \boldsymbol{r}$ and $\Delta \boldsymbol{p})$ and the quantum mechanical expectation value of energy $(\langle E\rangle)$ for two kinds of dissipations and analyzed them with respect to time $(t)$, damping parameters and flux parameter $(v)$. For the CK system, we observed that $\Delta \boldsymbol{r}(\Delta \boldsymbol{p})$ decreases (increases) with increasing $t$ and fixed $v$ and $\gamma$, indicating that the particle becomes more and more localized. Since the oscillating particle radiates, the motion is damped and the particle oscillates between two closer and closer points reducing $\Delta \boldsymbol{r}$.

On the other hand, as $v$ increases both $\Delta \boldsymbol{r}$ and $\Delta p$ increase for a fixed $t$. The flux parameter depends on the transversal area $A$ of the classical orbit. Therefore, the number of accessible points for the particle increases with increasing $A$ leading to an increase of $\Delta \boldsymbol{r}$. The increase of $\Delta p$ results from the fact that when the orbit radius increase, the particle velocity must increase to keep the orbital period constant.

By comparing the uncertainties $\Delta \boldsymbol{r}(t)$ for LE and CK dissipations in the range $t<2$, we observed that the $\Delta \boldsymbol{r}$ decreasing due to the LE dissipation is sharper than that for the CK one. By analyzing the system under the CK dissipation, we also observed that the flux parameter $v$ decreases the energy dissipation in a quantum dot for a certain range of $t$. This result can be useful to the improvement of quantum devices. A natural extension of this problem is the analysis of the quantum dynamics of a charged particle in an ion trap in the presence of dissipation.

\section{Acknowledgments}

The authors are grateful to the National Counsel of Scientific and Technological Development (CNPq) and to the National Council for the Improvement of Higher Education (CAPES) of Brazil for financial support.

\section{References}

[1] W. Ehrenburg and R.E. Siday, Proc. Phys. Soc. B 62, 8 (1949).

[2] Y. Aharonov and D. Bohm, Phys. Rev. 115, 485 (1959).

[3] R.G. Chambers, Phys. Rev. Lett. 5, 3 (1960).

[4] A. Tonomura, N. Osakabe, T. Matsuda, T. Kawasaki, J. Endo, S. Yano and H. Yamada, Phys. Rev. Lett. 56, 792 (1986).

[5] N. Okasabe T. Matsuda, T. Kawasaki, J. Endo, A. Tonomura, S. Yano and H. Yamada, Phys. Rev. A 34, 815 (1986).

[6] R.A. Webb, S. Washburn, C.P. Umbach, R.B. Laibowitz, Phys. Rev. Lett. 54, 2696 (1985).

[7] A. Bachtold, C. Strunk, J.P. Salvetat, J.M. Bonard, L. Forró, T. Nussbaumer and C. Schönenberger, Nature 397, 673 (1999).

[8] J. Cao, Q. Wang, M. Rolandi, H. Dai, Phys. Rev. Lett. 93, 216803 (2004).

[9] S. Russo, J.B. Oostinga, D. Wehenkel, H.B. Heersche, SS. Sobhani, L.M.K. Vandersypen and A.F. Morpurgo, Phys. Rev. B 77, 085413 (2008). 
[10] M. Huefner, F. Molitor, A. Jacobse, A. Pioda, C Stampfer, K. Ensslin and T. Ihn, Phys. Status Solidi B 246, 2756 (2009).

[11] M. Huefner, F. Molitor, A. Jacobsen, A. Pioda, C Stampfer, K. Ensslin and T. Ihn, New J. Phys. 12, 043054 (2010).

[12] P. Recher, B. Trauzettel, A. Rycerz, Y.M. Blanter, C.W.J. Beenakker and A.F. Morpurgo, Phys. Rev. B 76, 235404 (2007).

[13] C.S. Park, Phys. Lett. A 381, 1831 (2017).

[14] N. Ferkous and A. Bounames, Phys. Lett. A 325, 21 (2004).

[15] Y. Bouguerra, M. Maamache and A. Bounames, Int. J. Theor. Phys. 45, 1791 (2006).

[16] H.R. Lewis Jr. and W.B. Riesenfeld, J. Math. Phys. 10 1458 (1969).

[17] C.R. Hagen, Phys. Rev. Lett. 64, 53 (1990).

[18] C.R. Hagen, Int J. Mod. Phys. A 6, 3119 (1991).

[19] C.R. Hagen, Phys. Rev. D 64, 5935 (1993).

[20] F. Guinea, Phys. Rev. B 65, 205317 (2002).

[21] B. Horovitz and P. Le Doussal, Phys. Rev. B 74, 073104 (2006).

[22] A.O. Caldeira and A.J. Leggett, Phys. Rev. Lett. 46, 211 (1981).

[23] H. Dekker, Phys. Rep. 81, 1 (1981).

[24] N. Gisin, J. Phys. A: Math. Gen. 14, 2259 (1981).

[25] P. Caldirola, Nuovo Cim. 18, 393 (1941).

[26] E. Kanai, Prog. Theor. Phys. 3, 440 (1948).

[27] R.W. Hasse, J. Math. Phys. 16, 2005 (1975).

[28] D. Schuch, Phys. Rev. A 55, 935 (1997).

[29] S. Baskoutas, A. Janussis and R. Mignani, J. Phys. A: Math. Gen. 27, 2189 (1994).

[30] M. Tokieda and K. Hagino, Phys. Rev. C 95, 054604 (2017).

[31] D. Xu, J. Phys. A: Math. Gen. 35, L455 (2002).

[32] J.R. Choi, Phys. Scri. 73, 587 (2006).

[33] I.A. Pedrosa, A.P. Pinheiro, Prog. Theor. Phys. 125, 1133 (2011).

[34] I.A. Pedrosa, J.L. Melo and S. Salatiel, Eur. Phys. J. B 87, 269 (2014).

[35] V. Aguiar, I. Guedes and I.A. Pedrosa, Prog. Theor. Exp. Phys. 2015, 113A01 (2015).

[36] A. Dehghani, B. Mojaveri and R. Jafarzadeh Bahrbeig, Int. J. Theor. Phys. 58, 865 (2019).

[37] G. González, Int. J. Theor. Phys. 46, 417 (2007).

[38] S. González-Hernández, M.A. Ramírez-Moreno and G. Ares de Parga, Phys. A 516, 472 (2019).

[39] S. Baskoutas, C. Politis, M. Rieth and W. Schommers, Phys. A 292, 238 (2001).

[40] X.Y Pan, J.J. Zhu and Y.Q. Li, Phys. A 521, 293 (2019).

[41] A. Sulaiman, F.P. Zen, H. Alatas and L.T. Handoko, Phys. D 241, 1640 (2012).

[42] L.H. Yu and C.P. Sun, Phys. Rev. A 49, 592 (1994).

[43] C.P. Sun and L.H. Yu, Phys. Rev. A 51, 1845 (1995).

[44] W.E. Milne, J. Res. Natl. Bur. Stand. 43, 537 (1949).

[45] E. Pinney, Proc. Am. Math. Soc. 1, 681 (1950).

[46] I.A. Pedrosa, G.P. Serra and I. Guedes, Phys. Rev. A 56, 4300 (1997).

[47] S.F. Özeren, J. Math. Phys. 50, 012902 (2009).
[48] V.H.L. Bessa and I. Guedes, J. Math. Phys. 53, 122104 (2012).

[49] V. Aguiar and I. Guedes, Phys. A 401, 159 (2014).

[50] V. Aguiar and I. Guedes, Phys. Scr. 90, 045207 (2015). 